\title{
An Empirical Exploration of Phase Resetting for Robust Biped Locomotion with Dynamical Movement Primitives
}

\author{
Jun Nakanishi $^{* \dagger}$, Jun Morimoto*†, Gen Endo ${ }^{\ddagger *}$, Gordon Cheng ${ }^{* \dagger}$, Stefan Schaal ${ }^{\S *}$, and Mitsuo Kawato*† \\ * ATR Computational Neuroscience Laboratories, Kyoto, Japan \\ $\dagger$ ICORP, Japan Science and Technology Agency, Kyoto, Japan \\ $\ddagger$ Sony Corporation, Tokyo, Japan \\ $\S$ Dept. of Computer Science and Neuroscience, University of Southern California, Los Angeles, USA \\ \{jun,xmorimo,gendo,gordon\}@atr.jp, sschaal@usc.edu, kawato@atr.jp
}

\begin{abstract}
We propose a framework for learning biped locomotion using dynamical movement primitives based on nonlinear oscillators. In our previous work, we suggested dynamical movement primitives as a central pattern generator (CPG) to learn biped locomotion from demonstration. We introduced an adaptation algorithm for the frequency of the oscillators based on phase resetting at the instance of heel strike and entrainment between the phase oscillator and mechanical system using feedback from the environment.

In this paper, we empirically explore the role of phase resetting in the proposed algorithm for robust biped locomotion. We demonstrate that phase resetting contributes to robustness against external perturbations and environmental changes by numerical simulations and experiments with a physical biped robot.
\end{abstract}

\section{INTRODUCTION}

In our previous work [1], we proposed a method to learning biped locomotion from demonstration and its adaptation through coupling between the pattern generator and the mechanical system. We used dynamical movement primitives as a central pattern generator (CPG) of a biped robot, an approach we proposed for learning and encoding complex movements from human demonstration [2]. In the rhythmic dynamical movement primitives, kinematic movement plans are described in a set of nonlinear phase based oscillators, and demonstrated trajectories are learned using locally weighted regression. We introduced a frequency adaptation algorithm based on phase resetting [3] and entrainment between the phase oscillator and mechanical system using feedback from the environment. Numerical simulations demonstrated the effectiveness of the proposed control algorithm to achieve steady state walking and frequency adaptation of locomotion roughly at the natural frequency of the coupled system.

The objective of this paper is to empirically investigate the role of phase resetting in our control strategy with dynamical movement primitives for robustness of biped locomotion. In [1], we introduced phase resetting to our dynamical movement primitives motivated from a mathematical point of view as well as a biological perspective in order to achieve synchronization of the rhythm of oscillators with the timing of heel strike in biped locomotion. From a mathematical point of view, the phase resetting algorithm which we introduced can be interpreted as a discretized version of the synchronization mechanism of coupled phase oscillators. From a biological point of view, phenomena of phase resetting or phase shift are observed in many biological oscillators resulting from external perturbations, e.g., circadian pacemakers, biochemical oscillators and human finger tapping neural networks as mentioned in [3]. Phase resetting is related to the stability properties of neural rhythms, which can be analyzed by examining the phase dependent responses against perturbations. A recent work [4] studied functional roles of phase resetting for gait stability against external perturbations in biped locomotion. They investigated the behavior of a biped simulation model against external perturbations, where the phase of the prescribed joint trajectories obtained by Fourier fitting of humans' gait data are shifted in response to impulsive forces applied during walking. Their numerical simulations suggest that phase resetting helps maintain stability of periodic movements in biped locomotion in the presence of external perturbations. In [5], a similar approach of using coupled phase oscillators with phase resetting as a pattern generator for a biped locomotion robot was proposed. In their method, desired joint trajectories of the legs are generated from a nominal trajectory at the tip of each leg defined by a combination of simple prescribed functions of phase through inverse kinematics [5]. They experimentally demonstrated that the robot can adapt to a modest change in the angle of the slope from the level ground to a few degrees of an upslope. In comparison to [5], we believe that our method has the advantage of flexibility in encoding complex movements by imitation learning and the potential capability of improving learned movements through reinforcement learning [6].

In this paper, we evaluate robustness of walking with the proposed algorithm against external perturbations and environmental changes by numerical simulations and experiments with a physical biped robot. Note that the robot we use in this paper is an underactuated system having rounded soles with no ankles unlike many of the existing biped robots which have flat feet with ankle joint actuation. Numerical and experimental results demonstrate the effectiveness of phase resetting to achieve robust biped walking. 


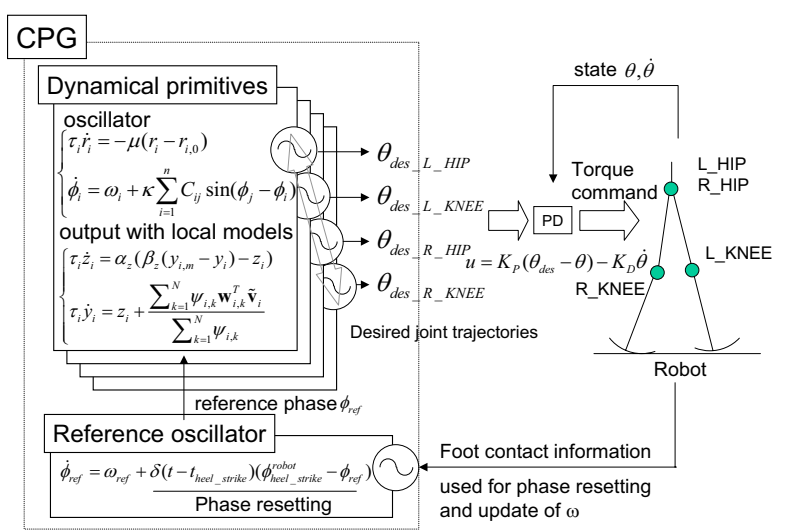

Fig. 1. Proposed control architecture for biped locomotion with dynamical movement primitives. The output of a dynamical movement primitive $y$ is used as the desired trajectory $\theta_{\text {des }}$ for each joint.
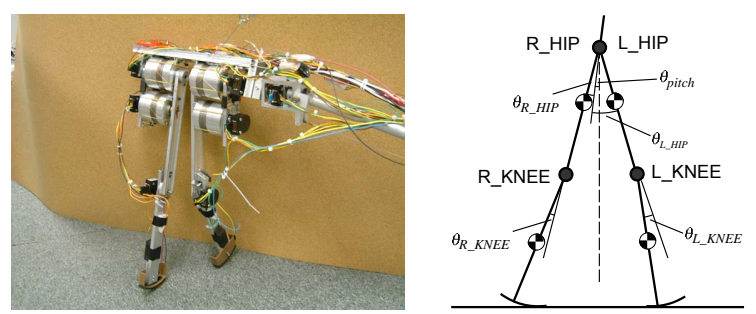

Fig. 2. Left: Physical system. Right: Five-link model of the robot.

\section{EXPERIMENTAL SETUP}

We use a planar 5-link biped robot developed in [7] (see Fig. 2). The height of the robot is $0.4 \mathrm{~m}$ and the weight is about $3.4 \mathrm{~kg}$. For numerical simulations, we use the following model: The length of each link of the leg is 0.2 $\mathrm{m}$. The mass of the body is $2.0 \mathrm{~kg}$, the thigh is $0.64 \mathrm{~kg}$ and the shank is $0.05 \mathrm{~kg}$.

The motion of the robot is constrained within the sagittal plane by a tether boom. The hip joints are directly actuated by direct drive motors, and the knee joints are driven by direct drive motors through a wire transmission mechanism with the reduction ratio of 2.0. These transmission mechanisms with low reduction ratio provide high back drivability at the joints. Foot contact with the ground is detected by foot switches. The robot is an underactuated system having rounded soles with no ankles. The robot is controlled with a real-time operating system, RT-Linux. The sampling frequency of the controller is $1 \mathrm{kHz}$.

\section{BIPED LOCOMOTION CONTROL WITH DYNAMICAL Movement PRimitives}

In this section, we outline the control framework for biped locomotion using dynamical movement primitives depicted in Fig. 1. In our initial work [1], we proposed an idea of using dynamical movement primitives as a CPG of a biped locomotion robot. In this paper, we extend the original CPG architecture in [1] by introducing coupling to oscillators in order to coordinate the desired phase relationship among the limbs of the robot.

\section{A. Rhythmic Dynamical Movement Primitives}

Rhythmic dynamical movement primitives encode periodic behavioral patterns as an output of a set of nonlinear dynamical systems composed of a canonical dynamical system with a phase oscillator and a transformation dynamical system with a nonlinear function approximator. We briefly review the formulation of the rhythmic movement primitives we proposed in [2].

Consider the following limit cycle oscillator characterized in terms of an amplitude $r$ and a phase $\phi$ as a canonical dynamical system which generates basic rhythmic patterns:

$$
\begin{aligned}
\dot{\phi} & =\omega \\
\tau \dot{r} & =-\mu\left(r-r_{0}\right)
\end{aligned}
$$

where $\omega$ is the frequency of the oscillator, $\tau$ is a temporal scaling factor defined by $\tau=1 / \omega, r_{0}$ determines the desired (relative) amplitude, and $\mu$ is a positive constant. When there are multiple oscillators, we will introduce coupling terms among the oscillators (see Section III-B.1). This rhythmic canonical system is designed to provide an amplitude signal $\tilde{\mathbf{v}}=[r \cos \phi, r \sin \phi]^{T}$ and phase variable $\bmod (\phi, 2 \pi)$ to the following second order transformation dynamical system $(z, y)$, where the output $y$ is used as the desired trajectory for the robot:

$$
\begin{aligned}
\tau \dot{z} & =\alpha_{z}\left(\beta_{z}\left(y_{m}-y\right)-z\right) \\
\tau \dot{y} & =z+f(\tilde{\mathbf{v}}, \phi)
\end{aligned}
$$

where $\alpha$ and $\beta$ are time constants, $y_{m}$ is an offset of the output trajectory. $f$ is a nonlinear function approximator using local linear models [8] of the form:

$$
f(\tilde{\mathbf{v}}, \phi)=\frac{\sum_{k=1}^{N} \Psi_{k} \mathbf{w}_{k}^{T} \tilde{\mathbf{v}}}{\sum_{k=1}^{N} \Psi_{k}}
$$

where $\mathbf{w}_{k}$ is the parameter vector of the $k$-th local model. Each local model is weighted by a Gaussian kernel function

$$
\Psi_{k}=\exp \left(-h_{k}\left(\bmod (\phi, 2 \pi)-c_{k}\right)^{2}\right)
$$

where $c_{k}$ is the center of the $k$-th linear model, and $h_{k}$ characterizes its width. A final prediction is calculated by the weighted average of the predictions of the individual models. The parameters $\mathbf{w}_{k}$ are determined by locally weighted learning [8] from a demonstrated trajectory $y_{\text {demo }}$. Given a sampled data point $\left(f_{\text {target }}, \tilde{\mathbf{v}}\right)$ at $t$ where

$$
f_{\text {target }}=\tau \dot{y}_{\text {demo }}-z_{\text {demo }}
$$

and

$$
\tau \dot{z}_{\text {demo }}=\alpha_{z}\left(\beta_{z}\left(y_{m}-y_{\text {demo }}\right)-z_{\text {demo }}\right),
$$

the learning problem is formulated to find the parameters $\mathbf{w}_{k}$ in (5) using incremental locally weighted regression technique [8] in which $\mathbf{w}_{i}$ is updated by

$$
\mathbf{w}_{k}^{t+1}=\mathbf{w}_{k}^{t}+\Psi_{k} \mathbf{P}_{k}^{t+1} \tilde{\mathbf{v}} e_{k}
$$

where

$$
\mathbf{P}_{k}^{t+1}=\frac{1}{\lambda}\left(\mathbf{P}_{k}^{t}-\frac{\mathbf{P}_{k}^{t} \tilde{\mathbf{v}} \tilde{\mathbf{v}}^{T} \mathbf{P}_{k}^{t}}{\frac{\lambda}{\Psi_{k}}+\tilde{\mathbf{v}}^{T} \mathbf{P}_{k}^{t} \tilde{\mathbf{v}}}\right)
$$




$$
e_{k}=f_{\text {target }}-\mathbf{w}_{k}^{T} \tilde{\mathbf{v}}
$$

and $\lambda \in[0,1]$ is a forgetting factor. As illustrated in [2], the amplitude, frequency and offset of the learned rhythmic patterns can be easily modified by scaling the parameters $r_{0}, \omega(=1 / \tau)$ and $y_{m}$ individually.

\section{B. Rhythmic Dynamical Movement Primitives as a CPG}

We use the rhythmic dynamical movement primitives mentioned above as a CPG for biped locomotion. Figure 1 illustrates the proposed control architecture in this paper. Each joint is equipped with a movement primitive which generates the desired joint trajectory. The output of the movement primitive $y$ is used as the desired trajectory $\theta_{\text {des }}$ for each joint. We define the index and the corresponding name of the joint as Left hip ( $i=1$, LHIP), and Left knee ( $i=2$, L_KNEE), Right hip ( $i=3$, R_HIP), and Right knee ( $i=4$, R_KNEE). An additional oscillator $\left(\phi_{\text {ref }}\right)$ is allocated to provide a reference phase signal to the limb oscillators, which is adjusted by the ground contact information at the instance of heel strike.

Section III-B.1 introduces coupling to the oscillators of the movement primitives to achieve the desired phase relationship between the limbs. Section III-B. 2 outlines the phase resetting and frequency adaptation algorithms of the learned periodic movements through the interaction among the coupled oscillators, robot and environment [1].

1) Inter- and Intra-limb Phase Coordination: In this paper, we introduce coupling among the oscillators to regulate the desired phase relationship between the limbs of the robot. This kind of coupling is motivated from a biological point of view where it has been hypothesized that coupling among neural oscillators plays an important role in coordinating the desired phase relationship of limb movements in locomotion and gait transition [9].

Consider the following coupling terms for the oscillator $i$

$$
\dot{\phi}_{i}=\omega_{i}+\kappa \sum_{i=1}^{N} C_{i j} \sin \left(\phi_{j}-\phi_{i}\right)
$$

where $\kappa$ is a positive constant gain, and $C_{i j}$ is an element of the $n \times n$ matrix $\mathbf{C}$ which characterizes the coupling with other oscillators. This form of coupling appears in various studies of coupled oscillators and their application, e.g., [5], [10], [11], [12], [13], [14]. Specifically, we employ the formulation in [10] to coordinate the desired phase relationship. We design the desired phase difference among the canonical oscillators such that the links of each leg move in phase (with zero phase difference), and the left and right legs move out of phase (with $\pi$ phase difference) by defining the phase of the oscillator as $\phi_{i}=0$ at the instance of heel strike of the corresponding leg. More specifically, we require $\phi_{1}-\phi_{2}=0, \phi_{3}-\phi_{4}=0, \phi_{1}-\phi_{3}=\pi$, and $\phi_{2}-\phi_{4}=\pi$. Thus, the connection matrix $\mathbf{C}$ is chosen to be

$$
\mathbf{C}=\left[\begin{array}{cccc}
0 & 1 & -1 & -1 \\
1 & 0 & -1 & -1 \\
-1 & -1 & 0 & 1 \\
-1 & -1 & 1 & 0
\end{array}\right]
$$

2) Phase Resetting and Frequency Adaptation of CPG: This section briefly outlines the phase resetting and frequency adaptation algorithms which we originally proposed in [1]. These algorithms were motivated by the synchronization mechanism of the coupled oscillators to adjust the frequency of the learned periodic motions by the robot through the interaction among the $\mathrm{CPG}$, robot and environment. In the original formualtion in [1], phase resetting was directly introduced to all the limb oscillators. Instead, in this paper, we first introduce phase resetting to the reference oscillator. Then, additional continuous coupling is introduced to the limb oscillators to achieve the desired relative phase to the reference oscillator in order to avoid discontinuity to the desired joint trajectories..

Consider the following phase resetting and frequency update law to the reference oscillator at the instance of heel contact:

$$
\begin{gathered}
\dot{\phi}_{\text {ref }}=\hat{\omega}_{\text {ref }}^{n}+\delta\left(t-t_{\text {heel strike }}\right)\left(\phi_{\text {heel strike }}^{\text {robot }}-\phi_{\text {ref }}\right) \\
\hat{\omega}_{\text {ref }}^{n+1}=\hat{\omega}_{\text {ref }}^{n}+K\left(\omega_{\text {measured }}^{n}-\hat{\omega}_{\text {ref }}^{n}\right)
\end{gathered}
$$

where $\delta$ is the Dirac's delta function, $n$ is the number of steps, and $\phi_{\text {heel strike }}^{\text {robot }}$ is the phase of the mechanical oscillator (robot) at heel strike defined as $\phi_{\text {heel }}^{\text {robtrike }}=0$ at the heel strike of the left leg, and $\phi_{\text {heel }}^{\text {robot }}$ strike $=\pi$ at the heel strike of the right leg. $\omega_{\text {measured }}^{n}$ is the measured frequency of locomotion defined by

$$
\omega_{\text {measured }}^{n}=\frac{\pi}{T_{\text {measured }}^{n}}
$$

where $T_{\text {measured }}^{n}$ is the stepping period of locomotion (half period with respect to the oscillator). At the same time, natural frequencies of all the limb oscillators $\omega_{i}$ are updated at the instance of heel contact such that $\omega_{i}=\hat{\omega}_{\text {ref }}^{n+1}$, and additional coupling is introduced to the limb oscillators with $\phi_{\text {ref }}$ to achieve the desired relative phase $\phi_{1}=\phi_{2}=$ $\phi_{\text {ref }}$ and $\phi_{3}=\phi_{4}=\phi_{\text {ref }}+\pi$.

\section{NUMERICAL SimUlations}

In this section, we numerically evaluate robustness of walking with the proposed algorithm against external perturbations by pushing the robot forward and backward with external forces during walking. Forces are applied for a duration of $0.1 \mathrm{sec}$ at different timing during a single step (at an interval of $0.1 \mathrm{rad}$ from 0 to $2 \pi$ of the phase of the reference oscillator). We judge that the robot could tolerate the perturbation if the robot continues to walk over 30 steps after the disturbance is applied. Fig. 3 shows the magnitude of the maximum external forces which the robot tolerated. When a forward perturbing force is applied, the robot could cope with up to $9.1 \mathrm{~N}(\max )$ at $\phi=1.1 \mathrm{rad}$, and $2.2 \mathrm{~N}$ (min) at $\phi=2.7 \mathrm{rad}$ of the perturbing forces. When a backward perturbing force is applied, the robot could cope with up to $-2.4 \mathrm{~N}(\max )$ at $\phi=4.9 \mathrm{rad}$ and $-1.0 \mathrm{~N}(\mathrm{~min})$ at $\phi=0.4$ and $\phi=0.5 \mathrm{rad}$ of the perturbing forces. In contrast, without phase resetting, the robot only could cope with much smaller disturbances, as indicated by the plot in Fig. 3, for example, the robot only tolerated up to 3.9 $\mathrm{N}$ of the forward perturbing force applied at $\phi=1.1$. On 


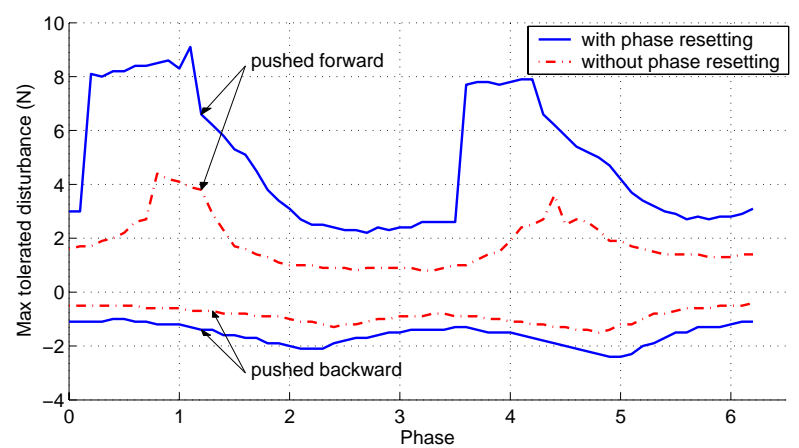

Fig. 3. Numerical evaluation of the robustness of walking against external perturbations. In the simulations, the robot is pushed forward and backward by perturbation forces for a duration of $0.1 \mathrm{sec}$ at difference timing during a single step. The plot shows the magnitude of the maximum external forces which the robot tolerated. This plot demonstrates that the robot could cope with much larger disturbances with phase resetting compared to the case without phase resetting.

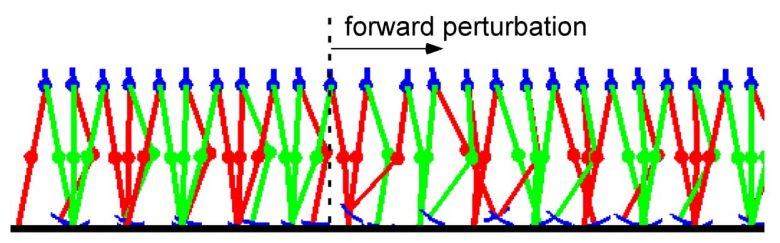

(a) with phase resetting

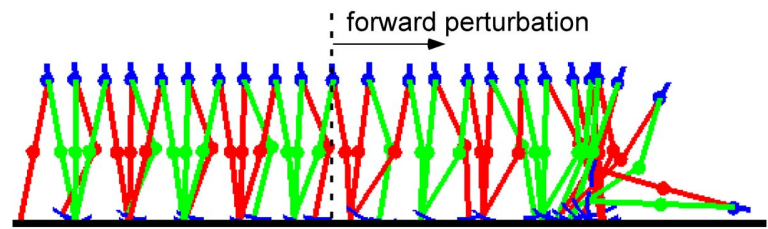

(b) without phase resetting

Fig. 4. An example of the response against forward perturbations. The robot is pushed forward by a perturbation force of $6.0 \mathrm{~N}$ for a duration of $0.1 \mathrm{sec}$ at $\phi=0.5$. (a) with phase resetting, the robot is able to reject the disturbance and continue to walk. (b) without phase resetting, the robot falls over after the perturbation is applied.

average, with phase resetting, the robot tolerated up to 4.83 $\mathrm{N}$ and $-1.58 \mathrm{~N}$ of the forward and backward perturbing forces respectively. However, without phase resetting, the robot tolerated only up to $1.79 \mathrm{~N}$ and $-0.88 \mathrm{~N}$ of the forward and backward perturbing forces respectively.

Fig. 4 depicts an example of the response against forward perturbations. The robot is pushed forward by a perturbation force of $6.0 \mathrm{~N}$ for a duration of $0.1 \mathrm{sec}$ at $\phi=0.5$. Fig. 5 depicts an example of the response against backward perturbations. The robot is pushed backward by a perturbation force of $-2.0 \mathrm{~N}$ for a duration of $0.1 \mathrm{sec}$ at $\phi=2.0$. The results in Fig. 4 (a) and Fig. 5 (a) show that with phase resetting, the robot is able to reject the disturbance and continue to walk. In contrast, the results in Fig. 4 (b) and Fig. 5 (b) show that without phase resetting, the robot falls over shortly after the perturbation is applied.

The simulation results demonstrate the effectiveness of phase resetting to achieve robust walking against disturbance.

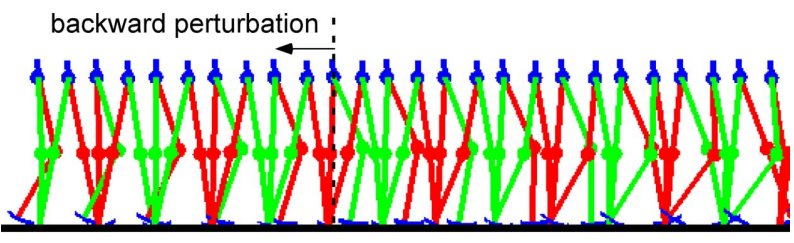

(a) with phase resetting

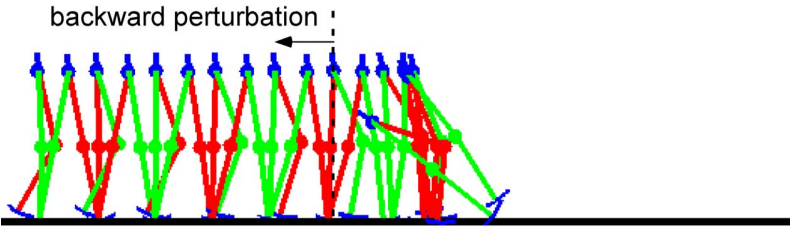

(b) without phase resetting

Fig. 5. An example of the response against backward perturbations. The robot is pushed backward by a perturbation force of $-2.0 \mathrm{~N}$ for a duration of $0.1 \mathrm{sec}$ at $\phi=2.0$. (a) with phase resetting, the robot is able to reject the disturbance and continue to walk. (b) without phase resetting, the robot falls over after the perturbation is applied.

\section{EXPERIMENTAL EVALUATIONS}

We implemented the proposed control framework on our biped robot. In the experimental implementation, our initial attempt to achieve biped locomotion using the human demonstrated trajectory was not successful. This was largely due to mechanical limitation of the experimental system and discrepancy in the ground contact condition between simulations and experiments. Thus, we used another target trajectory which was experimentally obtained from an actual trajectory of successful robot locomotion using a state machine controller. The state machine controller is designed to coordinate the leg movements with the physical state of the legged system based on the idea presented in [15]. To initiate locomotion in the experiments, we first suspended the robot with the legs swinging in the air, and then placed the robot on the ground manually. Thus, the initial condition of each run was not consistent, and occasionally the robot could not start walking or fell over after a couple of steps when the timing was not appropriate. In this experiment, the initial frequency of the oscillator was set to $\omega=5.71 \mathrm{rad} / \mathrm{s}$ (period of oscillation is $1.1 \mathrm{sec}$ ), and the adaptation gain in (12) was decreased according to an annealing procedure $K=\frac{K_{0}}{n}$, where $K_{0}=0.05$ and $n$ is the number of steps, as it is needed in most gradient descent procedure. We introduced an offset $\alpha$ for phase resetting

$$
\dot{\phi}_{\text {ref }}=\hat{\omega}^{n}+\delta\left(t-t_{\text {heel strike }}\right)\left(\phi_{\text {heel strike }}^{\text {robot }}-\phi_{\text {ref }}+\alpha\right)
$$

to adjust the timing of foot contact, where $\alpha$ is chosen to be $\alpha=0.8 \mathrm{rad}$. These parameters are determined empirically. Note that phase resetting with an offset effectively changes the period of oscillation. Fig. 6 presents snapshots of a walking experiment on a flat surface (carpet). Stepping period for a typical walking experiment was around $0.37 \mathrm{sec}$ as a result of frequency adaptation, and walking velocity was about $0.51 \mathrm{~m} / \mathrm{s}(1.87 \mathrm{~km} / \mathrm{h})$. 


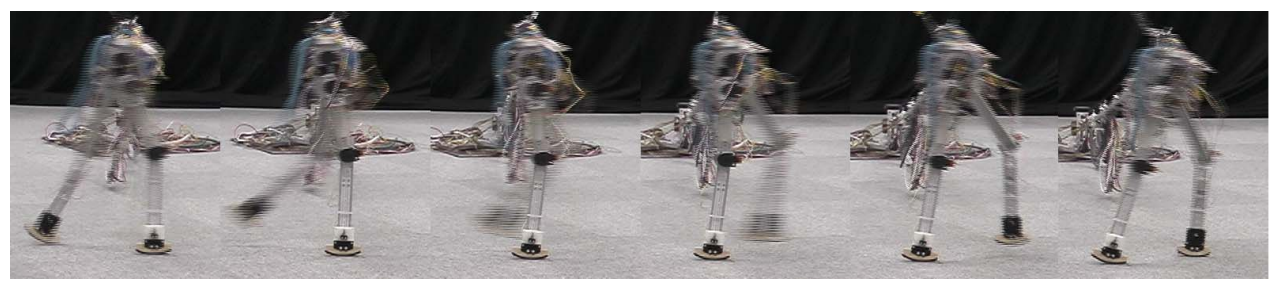

Fig. 6. Snapshots of a walking experiment on a flat surface (carpet) for a single step at 15 frames/sec $(1 \mathrm{frame} \approx 66 \mathrm{msec})$
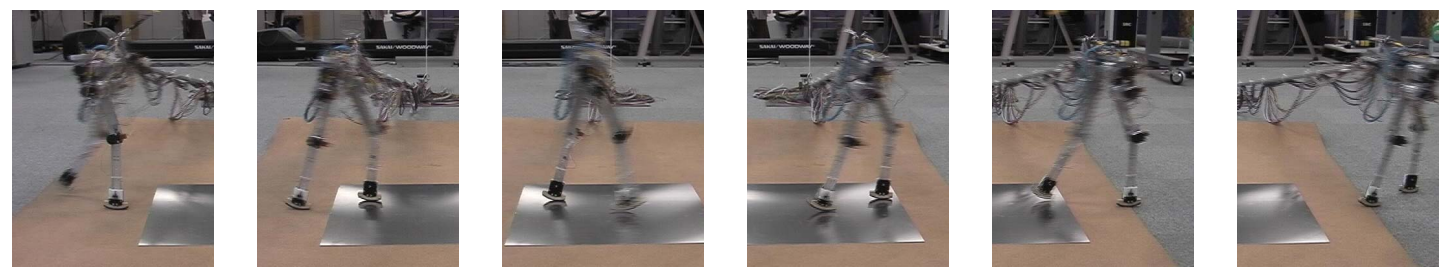

Fig. 7. Snapshots of a walking experiment over surfaces with different friction properties.
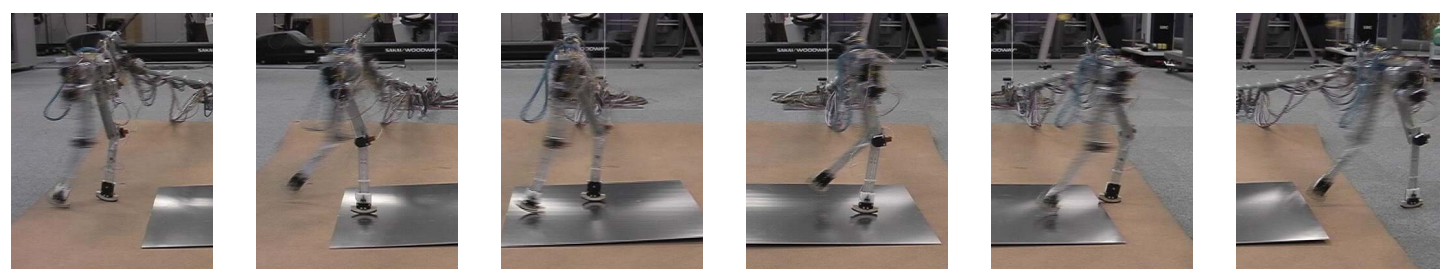

Fig. 8. Snapshots of a walking experiment over surfaces with different friction properties and a seesaw-like metal plate with a slight change in the slope. Also see the plots in Figs. 9-11 for joint trajectories, torque commands and stepping period for this experimental run, respectively.
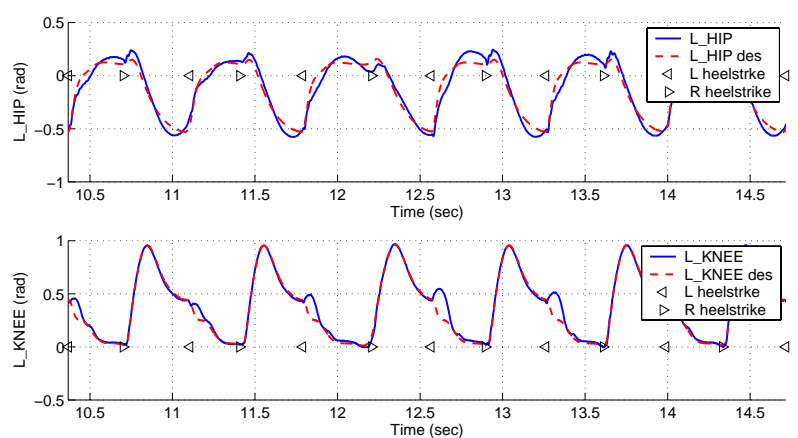

Fig. 9. Joint trajectories for the left leg and heel strike timing of the walking experiment over different surfaces with a see-saw like metal plate depicted in Fig. 8. These plots illustrate the joint trajectories of walking from 11th to 23rd steps in the corresponding plot in Fig. 11.

Robustness of the proposed algorithm is evaluated by testing walking over surfaces with different friction properties such as carpet, cork sheet (3 mm thick) and a metal plate $(2 \mathrm{~mm}$ thick). Fig. 7 shows an experimental result of walking over these different surfaces. In Fig. 8, the metal plate was placed so that the inclination of the slope slightly changes like a seesaw when the robot walks over it (the height of the center is $7 \mathrm{~mm}$ ). Figs. 9-11 show the desired and actual joint trajectories, the torque command, the timing of heel strike, and the stepping period of this walking experiment over a see-saw like metal
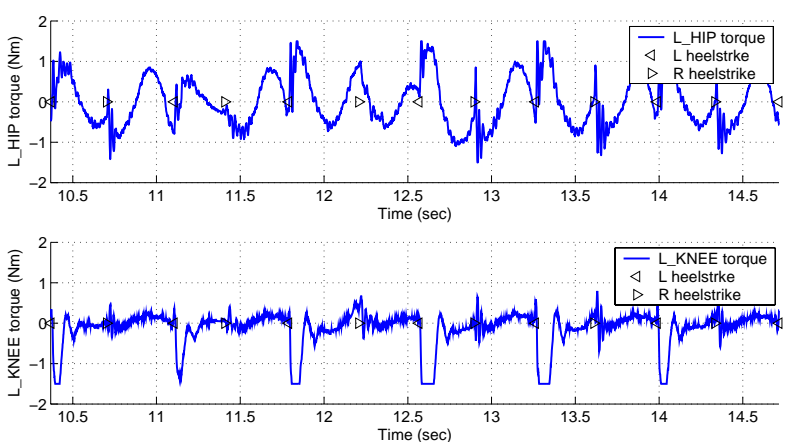

Fig. 10. Torque command to the left hip and knee joints of the walking experiment over different surfaces with a see-saw like metal plate depicted in Fig. 8.

plate respectively. The stepping period is disturbed when walking over the see-saw like plate (around 15th step, see Fig. 11), however, it is recovered after passing it. The robot could deal with these environmental changes as the experimental results in Figs. 7 and 8 demonstrate.

Note that even if we use the learned trajectory from the actual robot walking pattern, the robot could not walk by just replaying it as a desired trajectory. Phase resetting using foot contact information was necessary. This implies that appropriate on-line adjustment of the phase of the CPG by sensory feedback from the environment is essential to achieve successful locomotion. In addition, empirically 


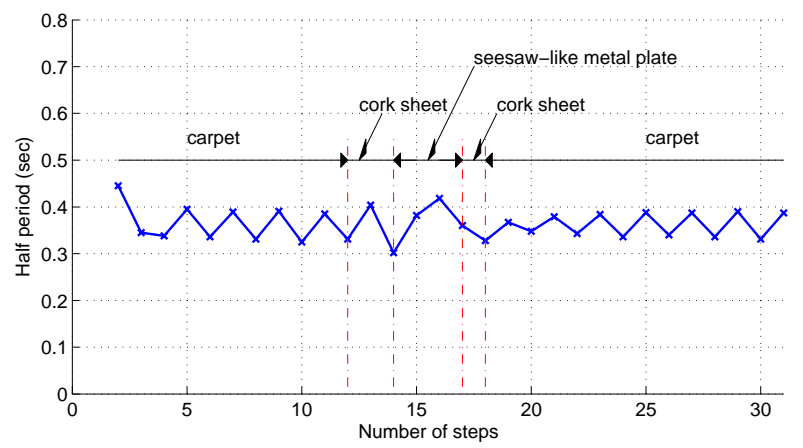

Fig. 11. Stepping period of a walking experiment over different surfaces with a see-saw like metal plate depicted in Fig. 8. The stepping period is disturbed when walking over the see-saw like metal plate, however, it is recovered after passing it.

we found that the proposed controller achieved much more robust walking compared to the state machine based controller which we originally designed.

\section{CONCLUSION}

In this paper, we empirically evaluated robustness of walking with the proposed algorithm against external perturbations and environmental changes. The numerical and experimental results demonstrate the effectiveness of phase resetting contributes to achieve robustness of walking against disturbances.

Future work will address initiation and termination of walking, and on-line balance compensation. We will also consider collection of human's walking data under various behavioral conditions. In our current study, we used a simple phase resetting mechanism in which the phase of the CPG is forced to be reset to a specific value at the instance of heel strike regardless of the current phase of the CPG. In the future, we are interested in the generalization of the idea of phase resetting to determine phase dependent reaction against external perturbations such as recovery from stumbling by designing an appropriate phase resetting curve [3]. Formal mathematical analysis will be required to understand the principle of periodic stability of a limit cycle solution to the dynamics of a combined oscillator and mechanical system.

\section{ACKNOWLEDGMENT}

This research was supported in part by National Science Foundation grants ECS-0325383, IIS-0312802, IIS0082995, ECS-0326095, ANI-0224419, a NASA grant AC\#98-516, an AFOSR grant on Intelligent Control, the Communications Research Laboratory of Japan, the ERATO Kawato Dynamic Brain Project funded by the Japan Science and Technology Agency, and the ATR Computational Neuroscience Laboratories.

\section{REFERENCES}

[1] J. Nakanishi, J. Morimoto, G. Endo, G. Cheng, S. Schaal, and M. Kawato, "Learning from demonstration and adaptation of biped locomotion with dynamical movement primitives," Workshop on Robot Programming by Demonstration, IEEE/RSJ International
Conference on Intelligent Robots and Systems, 2003, manuscript available at www. cns.atr.jp/ jun/int_pub.html.

[2] A. Ijspeert, J. Nakanishi, and S. Schaal, "Learning attractor landscapes for learning motor primitives," in Advances in Neural Information Processing Systems 15, S. Becker, S. Thrun, and K. Obermayer, Eds. MIT-Press, 2003, pp. 1547-1554.

[3] M. Kawato, "Transient and steady state phase response curves of limit cycle oscillators," Journal of Mathematical Biology, vol. 12 pp. 13-30, 1981

[4] T. Yamasaki, T. Nomura, and S. Sato, "Possible functional roles of phase resetting during walking," Biological Cybernetics, vol. 88, no. 6, pp. 468-496, 2003.

[5] K. Tsuchiya, S. Aoi, and K. Tsujita, "Locomotion control of a biped locomotion robot using nonlinear oscillaotrs," in Proceedings of the IEEE/RSJ International Conference on Intelligent Robots and Systems, 2003, pp. 1745-1750.

[6] J. Peters, S. Vijayakumar, and S. Schaal, "Reinforcement learning for humanoid robotics," in Proceedings of the Humanoids2003, Third IEEE-RAS International Conference on Humanoid Robots, 2003.

[7] J. Morimoto, G. Zeglin, and C. G. Atkeson, "Minimax differential dynamic programming: Application to a biped walking robot," in Proceedings of the IEEE/RSJ International Conference on Intelligent Robots and Systems, 2003, pp. 1927-1932.

[8] S. Schaal and C. G. Atkeson, "Constructive incremental learning from only local information," Neural Computation, vol. 10, no. 8, pp. 2047-2084, 1998.

[9] A. J. Ijspeert, "A connectionist central pattern generator for the aquatic and terrestrial gaits of a simulated salamander," Biological Cybernetics, pp. 331-348, 2001.

[10] E. Klavins and D. E. Koditschek, "Phase regulation of decentralized cyclic robotic systems," International Journal of Robotics Research, vol. 21, pp. 257-275, 2002.

[11] S. Ito, H. Yuasa, Z. wei Luo, M. Ito, and D. Yanagihara, "A mathematical model of adaptation in rhythmic motion to environmental changes," in Proceedings of the IEEE International Conference on Systems, Man and Cybernetics, 1997, pp. 275-280.

[12] K. Tsujita, K. Tsuchiya, and A. Onat, "Adaptive gait pattern control of a quadruped locomotion robot," in Proceedings of the IEEE/RSJ International Conference on Intelligent Robots and Systems, 2001, pp. 2318-2325.

[13] Y. Kuramoto, Chemical Oscillations, Waves, and Turbulence. Springer-Verlag, 1984.

[14] K. Sekiyama, J. Nakanishi, I. Takagawa, T. Higashi, and T. Fukuda, "Self-organizing control of urban traffic signal network," in Proceedings of the IEEE International Conference on Systems, Man and Cybernetics, 2001, pp. 2481-2486.

[15] J. K. Hodgins, "Biped gait transitions," in Proceedings of the IEEE International Conference on Robotics and Automation, 1991, pp. 2092-2097. 